University of Wollongong

Research Online

Faculty of Engineering - Papers (Archive)

Faculty of Engineering and Information

Sciences

$1-1-2010$

\title{
X-ray fluorescence imaging with the Medipix2 single-photon counting detector
}

J. Uher

CSIRO Process Science and Engineering

Gregory Harvey

University of Wollongong, gjh003@uow.edu.au

J. Jakubek

IEAP-CTU, Czech Republic

Follow this and additional works at: https://ro.uow.edu.au/engpapers

Part of the Engineering Commons

https://ro.uow.edu.au/engpapers/5020

\section{Recommended Citation}

Uher, J.; Harvey, Gregory; and Jakubek, J.: X-ray fluorescence imaging with the Medipix2 single-photon counting detector 2010, 1067-1073.

https://ro.uow.edu.au/engpapers/5020

Research Online is the open access institutional repository for the University of Wollongong. For further information contact the UOW Library: research-pubs@uow.edu.au 


\title{
X-Ray Fluorescence Imaging With the Medipix2 Single-Photon Counting Detector
}

\author{
J. Uher, G. Harvey, and J. Jakubek
}

\begin{abstract}
Material-resolved X-ray imaging or colour X-ray imaging is of a great interest for many applications ranging from physics, industry to medicine and biology. X-ray fluorescence offers a method for producing such images if the energies and positions of origin of the fluorescent photons can be adequately resolved.

This paper describes application of the Medipix2 single photon counting imaging detector $\left(256 \times 256\right.$ pixels each of $55 \times 55 \mu \mathrm{m}^{2}$ size) for this purpose.

The basics of the method are explained including details of the energy calibration of all $65 \mathrm{k}$ individual pixels. The effect of charge sharing is discussed and a method for its characterisation based on numerical calculation presented. The charge sharing calculation is then used to generate the Medipix2 detector response matrix, which is subsequently used for analysis of measured spectra in detector pixels.
\end{abstract}

Index Terms-Charge sharing, imaging, Medipix, photon counting, pixel detector, $\mathrm{X}$-ray fluorescence.

\section{INTRODUCTION}

$\mathbf{X}$ -RAY FLUORESCENCE IMAGING (XRFI) is a powerful tool to study spatial distribution of elements in a wide variety of samples and applications. Most advanced are beam scanning devices that utilize either synchrotron beams, proton beams [1] or micro-focus X-ray tubes coupled with $\mathrm{X}$-ray optics [2]. However, these approaches require either complex beamlines, or a precise alignment of the X-ray optics and mechanics for scanning with the sample.

Another approach is based on the full-field imaging. The primary X-ray source irradiates the whole sample and the secondary X-ray fluorescent photons are projected through a pinhole onto an energy sensitive CCD [3]. Such a device does not require any beam-scanning mechanism. The limitation is in sensitivity and readout speed of the CCD sensor. For instance, the device used in [3] has only $80 \mu \mathrm{m}$ thick sensitive Silicon volume. Moreover, in order to measure the X-ray energy, each pixel must not see more than one X-ray photon during the ex-

Manuscript received November 14, 2010; revised March 10, 2011, August 16, 2011; accepted October 10, 2011. Date of publication December 26, 2011; date of current version February 10, 2012

J. Uher is with CSIRO Process Science and Engineering, Lucas Heights, NSW, Australia and also with CSIRO Minerals Down Under National Research Flagship, Lucas Heights, NSW, Australia (e-mail: josef.uher@csiro.au).

G. Harvey is with Centre for Medical Radiation Physics, University of Wollongong, Wollongong 2522, NSW, Australia (e-mail: gregory.harvey@csiro. au).

J. Jakubek is with IEAP-CTU, Horska 3a/22, Prague, Czech Republic (e-mail: jan.jakubek@utef.cvut.cz).

Color versions of one or more of the figures in this paper are available online at http://ieeexplore.ieee.org.

Digital Object Identifier 10.1109/TNS.2011.2173352 posure. Therefore, the exposure time has to be short. That, together with the readout time of the whole CCD matrix (10 s), contributes significantly to the dead time and increases the required total measurement time.

Our approach to the XRFI method is also based on a pinhole, but in combination with X-ray single photon counting imaging detectors Medipix [4] with energy discrimination capabilities. The Medipix detectors offer a higher X-ray detection sensitivity thanks to their thicker sensors (typically $300 \mu \mathrm{m}$ ). There is also possibility to use CdTe sensors in the future to further improve the sensitivity to higher energies of X-rays [5]. The final result will be an X-ray camera that can be used to analyze (parts of) large stationary samples in cases where moving the sample or detector/source is not possible and hence the scanning XRFI techniques are not applicable. This paper presents our first results obtained in a laboratory setup and focuses on details of characterization and calibration of the detector.

We used the Medipix2 MXR pixel detector [4] in our prototype. It is a silicon imaging detector with $256 \times 256$ pixels, each of $55 \times 55 \mu \mathrm{m}^{2}$ size. The total sensor area is $1.4 \times 1.4 \mathrm{~cm}^{2}$. The silicon sensor is $300 \mu \mathrm{m}$ thick and, is bump-bonded to an ASIC readout chip. All pixels have their own electronics, consisting of an amplifier, energy discriminators and a counter. The energy discrimination allows Medipix2 to be used as an XRFI detector for material-resolved imaging. It was shown that elements different by one atomic number could be resolved [6] with Medipix2.

The XRFI test setup is shown in Fig. 1. It contained a Hamamatsu X-ray tube, the Medipix2 detector and a lead pinhole. The Medipix device was entirely shielded by a lead encapsulation to prevent X-rays scattered inside the cabinet from entering the detector. The sample was irradiated with primary X-rays from the tube. The primary X-ray beam generated in the sample X-ray fluorescent photons that were projected onto the Medipix2 detector through the pinhole.

The spatial resolution of the XRFI method is primarily determined by the pinhole diameter [3]. A larger pinhole is needed to achieve sufficient statistics for images in a reasonably short time (minutes, hours); however, this negatively affects the spatial resolution of the image.

In order to resolve elements with close X-ray emission lines, the full X-ray spectrum has to be measured in each Medpix2 pixel. The spectrum was obtained by running a threshold scan; i.e., by varying the low energy threshold across the range of anticipated X-rays energies. An integral X-ray spectrum was thus measured in each pixel.

The data analysis technique described in [6] is based on spectrum decomposition and requires premeasured X-ray fluores- 

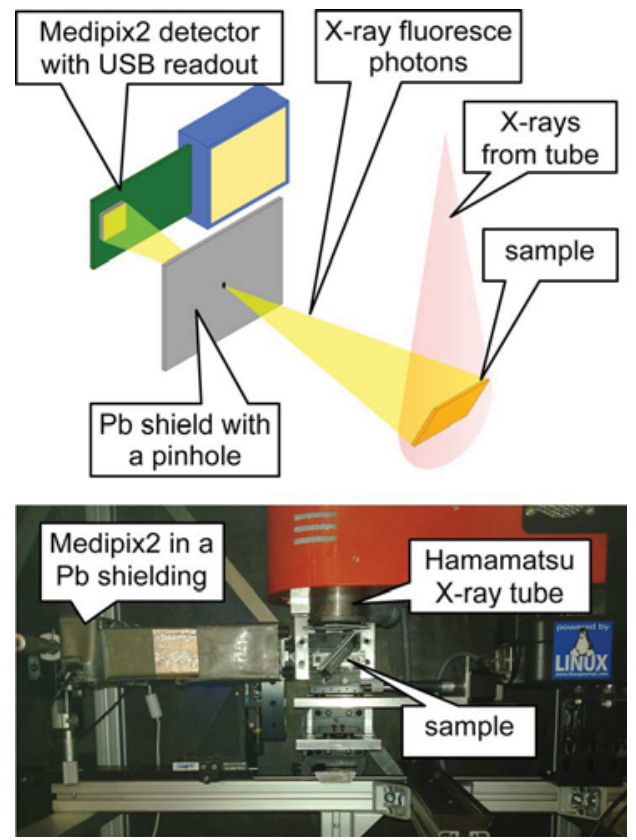

Fig. 1. Schematics and photo of the X-ray fluorescence experimental setup.

TABLE I

ELEMENTS AND Their MAJOR X-RAY Fluorescent Lines USED IN THE Calibration and in Tests of the XRFI. The Data Were Obtained From THE NIST DATABASE [8]

\begin{tabular}{ccccc} 
Element & $\begin{array}{c}\mathrm{K} \alpha_{1} \\
{[\mathrm{keV}]}\end{array}$ & $\begin{array}{c}\mathrm{K} \alpha_{2} \\
{[\mathrm{keV}]}\end{array}$ & $\begin{array}{c}\mathrm{K} \beta_{1} \\
{[\mathrm{keV}]}\end{array}$ & $\begin{array}{c}\mathrm{K} \beta_{2} \\
{[\mathrm{keV}]}\end{array}$ \\
\hline $\mathrm{Ge}$ & 9.887 & 9.856 & 10.984 & 11.101 \\
$\mathrm{Zr}$ & 15.775 & 15.691 & 17.667 & 17.971 \\
$\mathrm{Mo}$ & 17.479 & 17.374 & 19.607 & 19.967 \\
$\mathrm{Pd}$ & 21.177 & 21.021 & 23.820 & 24.302 \\
$\mathrm{Ag}$ & 22.162 & 21.991 & 24.943 & 25.458 \\
$\mathrm{Sn}$ & 25.271 & 25.044 & 28.486 & 29.109
\end{tabular}

cence (XRF) spectra of elements anticipated in the examined sample. This requirement limits the practicality of the method, as it is difficult to generate a comprehensive database of spectral responses. This paper presents a technique for calculating the Medipix2 response to different X-ray fluorescence photon energies and shows its application to resolving materials in the XRF image.

\section{Per-PiXel EnERgy CALIBration}

The analysis of X-ray spectra measured by the Medipix detector requires energy calibration of all $65 \mathrm{k}$ pixels individually. Energy calibration of Medipix 2 has been performed previously using X-rays and gamma rays [7].

The experimental setup utilized for the energy calibration measurement was the same as for the XRF imaging shown in Fig. 1. The calibration employed X-ray fluorescent photons. The list of elements and emission lines used for the calibration and experiments described in this paper is shown in Table I.

The primary X-ray beam was filtered by $50 \mu \mathrm{m}$ of Tungsten to reduce the flux of low energy photons that would be otherwise scattered from the sample into the detector. The voltage of the primary X-ray beam was $100 \mathrm{kVp}$ and the current was $200 \mu \mathrm{A}$.
The target of the X-ray tube was Tungsten. The sample-to-pinhole distance was $9 \mathrm{~cm}$ and the distance between the pinhole and the Medipix 2 was $15 \mathrm{~cm}$. The purpose of the pinhole during the calibration was to reduce amount of the scattered radiation reaching the detector from inside of the X-ray cabinet. A large pinhole $(\emptyset 4.5 \mathrm{~mm})$ was chosen. The large pinhole sufficiently suppressed the scattered radiation while facilitated higher count rates of detected photons emitted from the calibration material, which was necessary for a reliable calibration. An integral spectrum of fluorescent photons for $\mathrm{Ge}, \mathrm{Zr}$, Pd and $\mathrm{Sn}$ was measured by scanning the lower discrimination threshold.

The device contains 4-bit coarse threshold Digital-To-Analogue Converter (DAC) and 10-bit fine DAC. Only the fine 10-bit DAC was used for the threshold scanning. The threshold DAC step was 2, which corresponds in average to step of $0.3 \mathrm{keV}$ in an individual pixel. The device provides also an upper threshold. However, it was not used in the measurement. Utilization of the upper threshold and therefore scanning with an energy window would require a separate energy calibration of the upper threshold and adding extra complexity to the data analysis (for example correcting on change of the energy window width during the scan).

The exposure time was $200 \mathrm{~s}$ for each step. The overall scan thus took between 2.5 and 5 hours. The applied bias was $100 \mathrm{~V}$.

Differentiation of the measured integral spectrum on a perpixel basis means subtraction of large numbers with a small difference. That leads to a large statistical error. Therefore, the integral spectrum was fitted rather than the differential one, using Gaussian peak and linear background:

$$
y(x)=\int_{\mathrm{THL}_{1}}^{\mathrm{THL}_{2}}\left[a e^{\frac{-\left(x-x_{0}\right)^{2}}{2 \sigma^{2}}}+b+c x\right] d x,
$$

where $a, b, c, x_{0}$ are parameters obtained from the fit. The mean value $x_{0}$ corresponds to the position of $\mathrm{K} \alpha_{1}$ line. $\mathrm{K} \alpha_{2}$ lines of elements used in the experimental study were too close to $\mathrm{K} \alpha_{1}$ lines and could not be resolved by the detector due to its limited energy resolution. The $\mathrm{K} \alpha_{2}$ lines where therefore neglected. The non-zero counts in Fig. 2 left to the step that corresponds to the $\mathrm{K} \alpha_{1}$ peak are caused by $\mathrm{K} \beta_{1,2}$ photons. However, only the intense $\mathrm{K} \alpha_{1}$ was used for the calibration and the $\mathrm{K} \beta_{1,2}$ lines were considered as a background. The parameter $\sigma$ was estimated from the total spectrum that was constructed as a sum of spectra measured in individual pixels. The values THL1 and THL2 are limits of the fit range expressed in values of the DAC that sets the threshold for all pixels. An example of a fit in one pixel is in Fig. 2.

The distribution of Palladium $\mathrm{K} \alpha_{1}$ peak positions in all pixels is shown in Fig. 3. The spectrum in Fig. 3 was calibrated with an average calibration determined from the total spectra generated by sum of individual spectra in all pixels. The following Fig. 4 shows the same distribution after the per-pixel energy calibration. Post-calibration, the sigma of the distribution dropped from 0.62 to $0.22 \mathrm{keV}$. The inset of Fig. 3 reveals the non-uniformity of the detector parameters (corresponding to the asymmetrical shape of the distribution). The per-pixel energy calibration 


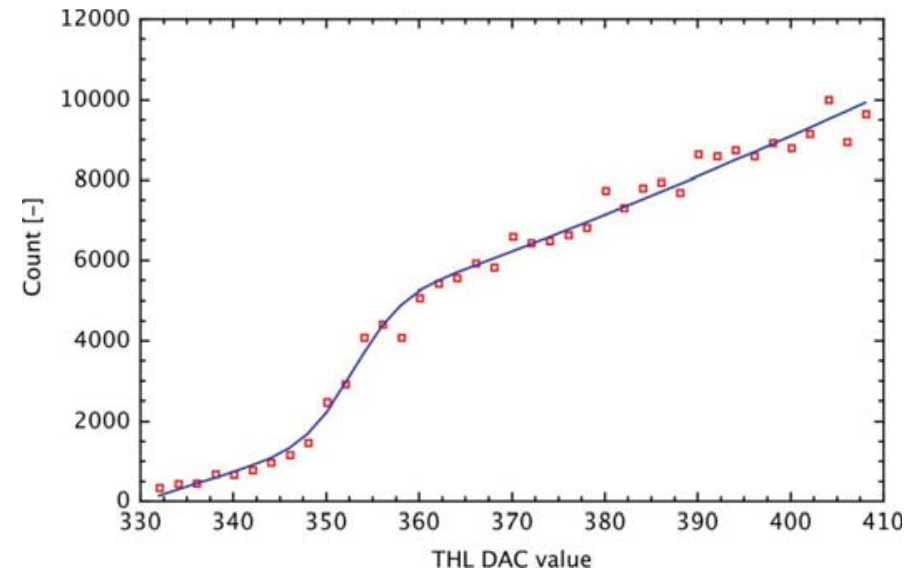

Fig. 2. Example of the measured integral spectrum of the Pd X-ray fluorescence (squares) fitted with function from (1) (solid line) to determine the peak position. The $\mathrm{x}$-axis shows the values of Digital-To-Analogue Converter of the energy threshold (THL). The lower the THL value, the higher the threshold energy; therefore, the spectrum is mirrored (the lower energies of photons are at higher values of THL).

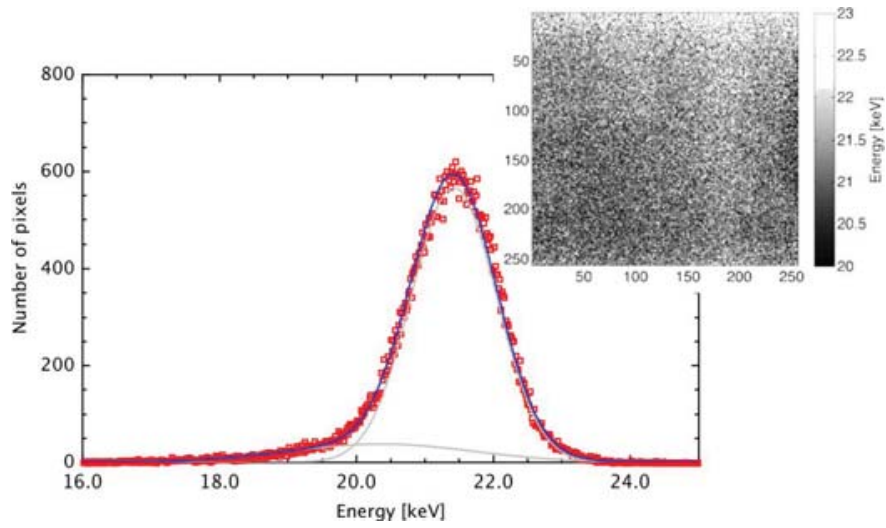

Fig. 3. Distribution of the fitted positions of the $\mathrm{K} \alpha_{1}$ line of $\mathrm{Pd}$ in individual pixels (squares). The distribution shown is fitted with sum of two Gaussians (solid lines show also each of the two Gaussians in the sum) with $\sigma_{1}=0.62$ and $\sigma_{2}=1.34 \mathrm{keV}$. The inset shows the spatial distribution of the peak positions in pixels.

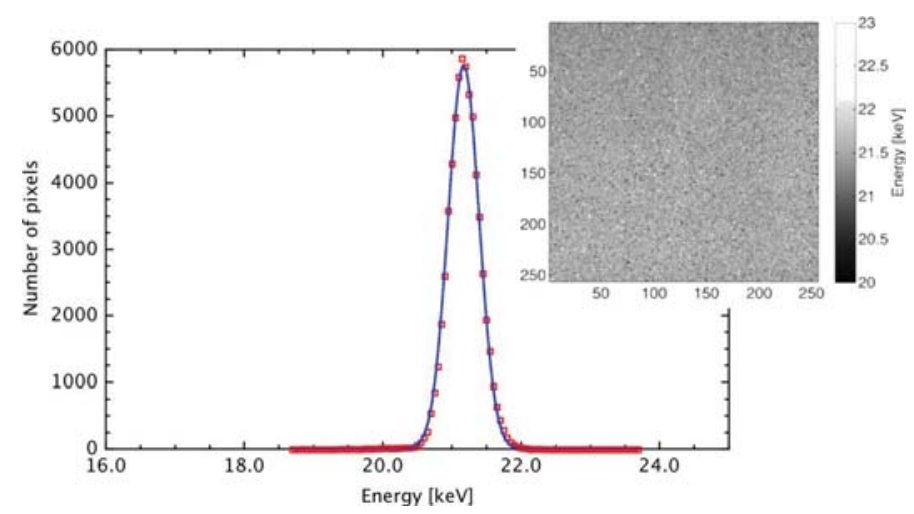

Fig. 4. The distribution of fitted positions (squares) of the $\mathrm{K} \alpha_{1}$ line of $\mathrm{Pd}$ from Fig. 3 after the per-pixel calibration. The solid line is a fit with Gaussian function. The right tail of the distribution in Fig. 3 was corrected by the per-pixel calibration. The sigma of the distribution after the calibration is $\sigma_{1}=0.22 \mathrm{keV}$. The inset shows that the spatial non-uniformity was improved as well.

corrects on this non-uniformity across the chip, as shown in the inset of Fig. 4.

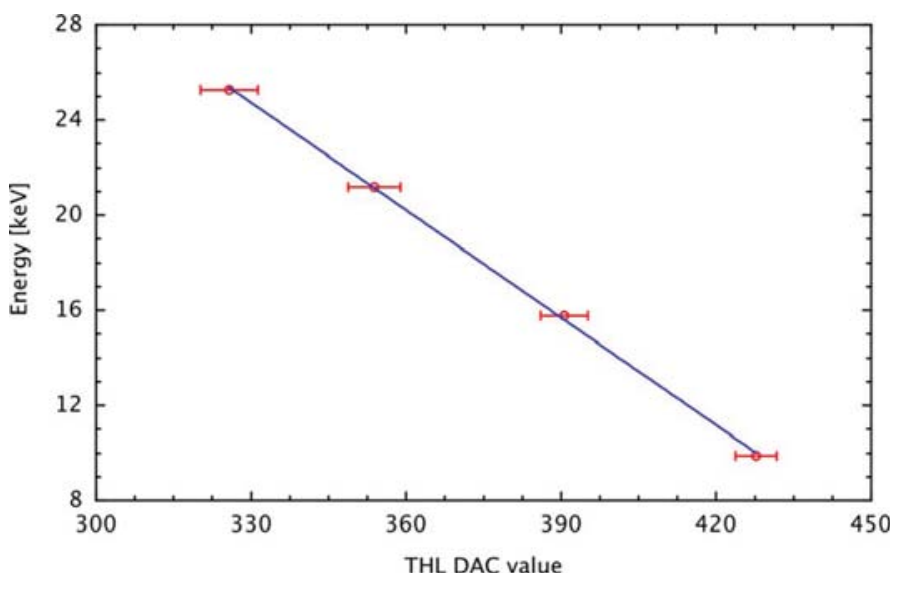

Fig. 5. Example of energy calibration of one selected pixel. The measured data (circles) were fitted with a linear function (solid line).

An example of one linear calibration fit is shown in Fig. 5. For the majority of all pixels the THL value varied linearly with energy. Only about $0.6 \%$ of all $65 \mathrm{k}$ pixels had to be masked typically due to an increased noise that prevented finding the positions of the XRF peaks for the calibration.

\section{CHARge Sharing}

An effect inside a pixel detector that complicates the measured X-ray fluorescent spectra is the charge sharing which occurs between pixels. As the size of pixels is $55 \times 55 \mu \mathrm{m}^{2}$ and the detector thickness is $300 \mu \mathrm{m}$, the charge generated by an X-ray photon spreads to more than one pixel during the charge collection process [9]. This leads to a low energy tail in the measured spectrum.

The total integral spectra (sum of spectra for individual pixels measured by the threshold scan) of elements in the calibration were differentiated and used to study the charge sharing effect.

The Fig. 3 shows that the true value of the threshold varies from pixel to pixel. A direct summation of the spectra from individual pixels would lead to a degradation of the energy resolution within the spectrum. Therefore, each individual pixel spectrum was energy calibrated; these calibrated spectra were then summed and differentiated. The resulting spectra are shown in Figs. 6 to 9. Starting from $\mathrm{Zr}$ (Fig. 7), it is possible to resolve the $K \beta_{1}$ lines. The left tail in the spectra caused by the charge sharing effect is visible in all shown images. The low energy tails of the peaks did not present problem in the energy calibration fits, as they could be sufficiently described in the proximity of the peak using the linear part of (1). However, a complex spectrum with number of peaks has to be analyzed at the full range of energies. The charge sharing effect must be characterized more precisely in order to reconstruct the original spectrum of the incoming radiation.

The treatment of charge sharing has previously been described in literature. Researches in [10] performed a full Monte-Carlo simulation of X-ray photon interactions with the Medipix sensor and the subsequent charge collection. Another simpler approach using an analytical calculation was published in [9]. The precise model presented in [10] provides better agreement in the charge-sharing region, compared to the simple 


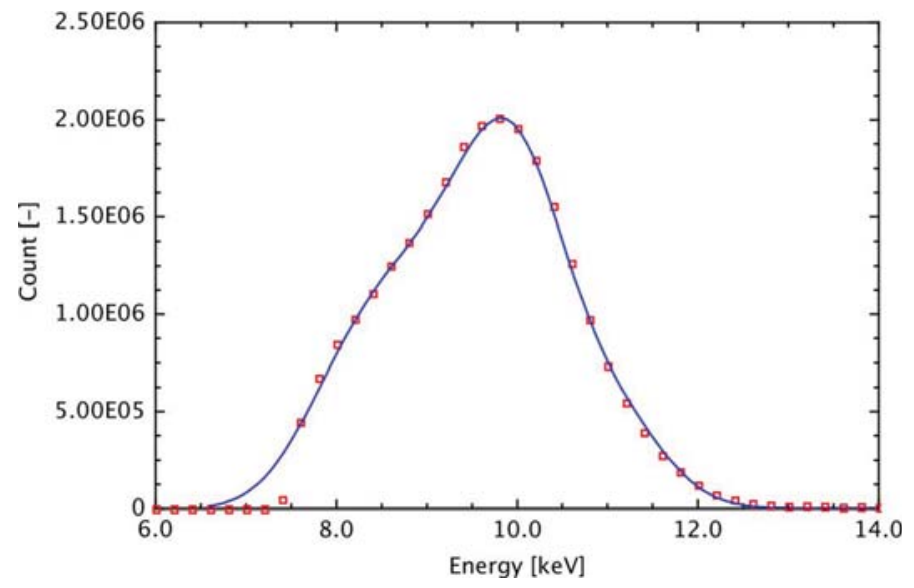

Fig. 6. Peak of Ge X-ray fluorescence. The squares represent the measured data and the full line is the fit using the charge sharing calculation.

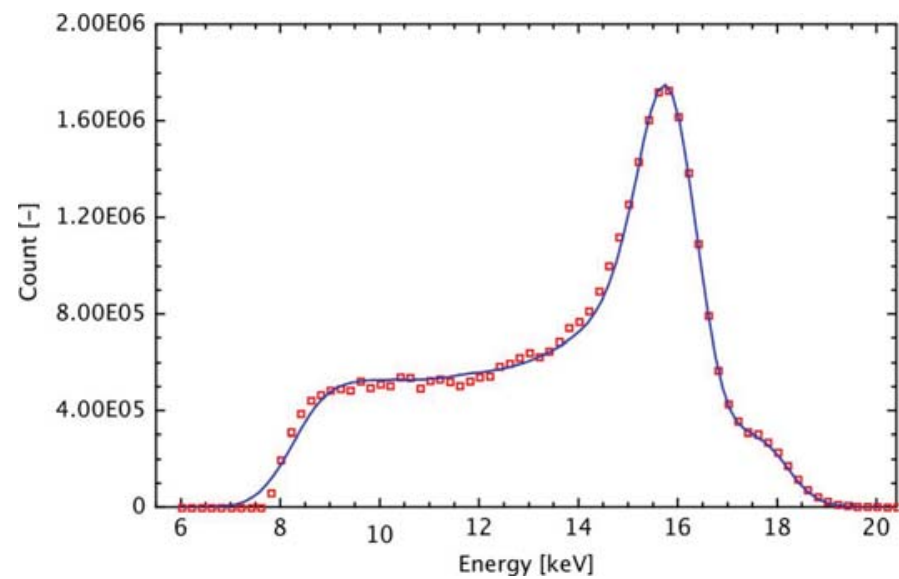

Fig. 7. Peak of $\mathrm{Zr}$ X-ray fluorescence. The $\mathrm{K} \beta_{1}$ line at $17.667 \mathrm{keV}$ becomes visible. The squares represent the measured data and the full line is the fit using the charge sharing calculation.

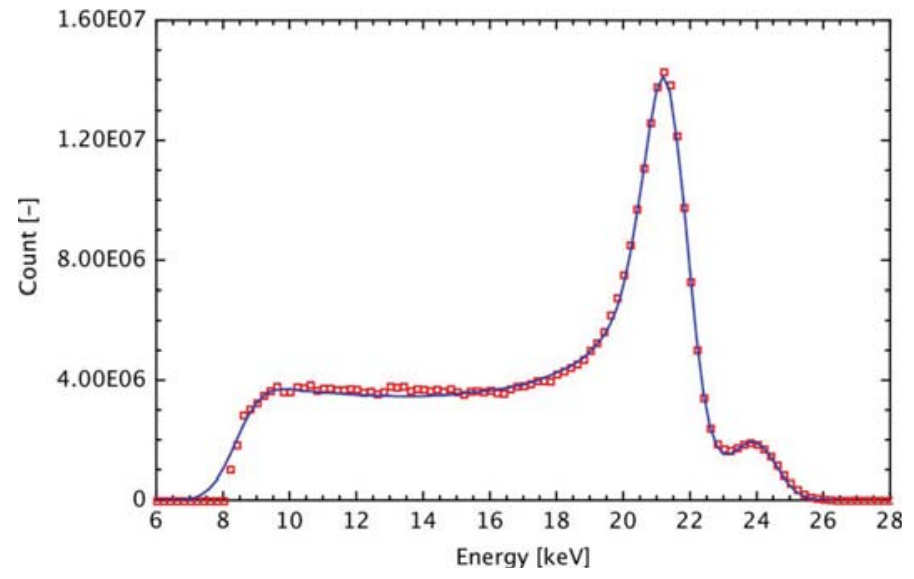

Fig. 8. Peak of PdX-ray fluorescence. The squares represent the measured data and the full line is the fit using the charge sharing calculation.

model described in [9], at the cost of complexity of the simulation. We have attempted to combine the advantages of both approaches: the high accuracy of the Monte-Carlo simulation with simplicity and speed of the analytical model of charge sharing.

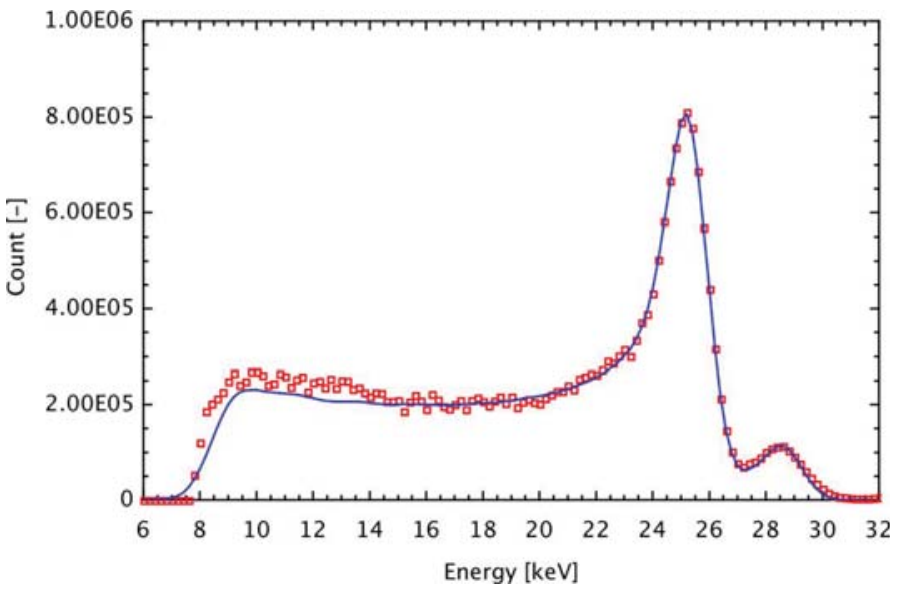

Fig. 9. Peak of Sn X-ray fluorescence. The squares represent the measured data and the full line is the fit using the charge sharing calculation.

The charge sharing calculation presented here was based on experimental results. The charge collection in a pixelated silicon sensor was extensively studied with the Timepix detector [12], [13], [15]. The Timepix detector is a descendant of the Medipix2 device. It has the same size and number of pixels, but contrary to Medipix2, it allows charge collected in each individual pixel to be measured. Therefore, it can serve as a tool for the charge collection characterization. The pixel pitch (55 $\mu \mathrm{m}$ ), the applied bias (typically $100 \mathrm{~V}$ ), and the sensor thickness $(300 \mu \mathrm{m})$ are the same as in the case of Medipx2. Therefore, the results of measurement with the Timepix device are also valid for the Medipix2. The two important conclusions of the experimental studies were that the charge spread can be described with a 2D Gaussian distribution [14], which was in fact just a confirmation of theory [15] and other experimental evaluations [10]. The second important conclusion was that the width of the Gaussian, and therefore also the cluster ${ }^{1}$ size, as discussed in [12], was changing almost linearly with the depth of photon interaction.

It should be noted that the disadvantage of the Timepix device for spectral measurement is currently its readout speed. The spectra are reconstructed from individual clusters of pixels with signal generated by the incoming radiation. To analyze the detected clusters correctly, cluster overlaps must be avoided. That requires short exposure times. The Timepix readout architecture is the same as in the case of Medipix2 device. Therefore, the whole detector matrix must be read out for each frame that contains only few photon hits. The frame exposure time has to be as low as $100 \mu \mathrm{s}$. However, the USB readout allows collecting only about 4 frames/s and that leads to a significant dead time [12]. Whereas, the exposure time of one step of the threshold scan in the Medipix2 device can be long (hundreds of seconds) to accumulated sufficient statistics. The slow readout has then only a negligible effect. Therefore, the Medipix 2 still remains a device relevant for the energy sensitive imaging application.

The effect of the charge sharing on the X-ray spectrum measured with the Medipix2 can be calculated as follows. Let the Silicon sensor be divided into $L$ layers and assume that the

\footnotetext{
${ }^{1}$ The charge spreads to neighboring pixels and therefore a single particle
} (photon) creates signal in a "cluster" of adjacent pixels 


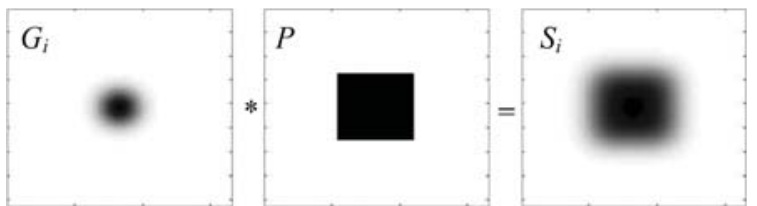

Fig. 10. Illustrative graphical representation of functions that appear in the convolution in (2).

spread of the charge is limited to an area of $3 \times 3$ pixels. This assumption is appropriate because the observed cluster size for the anticipated energy range $(\sim 7$ to $\sim 30 \mathrm{keV})$ was less than 3 pixels [12] for the applied bias of $100 \mathrm{~V}$. For $i$-th layer we calculate convolution:

$$
S_{i}=G_{i} * P
$$

where:

$$
G_{i}(x, y)=\frac{e^{-\frac{\left(x-x_{0}\right)^{2}+\left(y-y_{0}\right)^{2}}{2 \sigma_{i}^{2}}}}{\iint_{A} e^{-\frac{\left(x-x_{0}\right)^{2}+\left(y-y_{0}\right)^{2}}{2 \sigma_{i}^{2}}} d x d y},
$$

is the $2 \mathrm{D}$ Gaussian describing the spread of charge for the photon interaction in the $i$-th layer of the sensor. Parameters $x_{0}$ and $y_{0}$ are the center of the $3 \times 3$ pixel array. The function $G_{i}$ is normalized by its integral over the area $A$ of the $3 \times 3$ pixel array. The parameter $\sigma_{i}$ represents the size of the charge spread and it is calculated as a linear interpolation between values in the top ( $\mathrm{i}=1, \mathrm{X}$-ray entrance window) and bottom $(\mathrm{i}=\mathrm{L})$ layer of the detector:

$$
\sigma_{i}=\left(\sigma_{i=L}-\sigma_{i=1}\right) \frac{i-1}{L-1}+\sigma_{i=1}
$$

For the parameters $\sigma_{i=1}, \sigma_{i=L}$ applies $\sigma_{i=1}>\sigma_{i=L}$, i.e., the charge spreads more if the photon is absorbed on the top of the detector and it has to travel across the whole detector thickness before it is collected by a pixel electrode. The function $P$ represents the central pixel of interest for which the charge sharing (i.e., the detected spectrum) is calculated:

$$
\begin{aligned}
& P(x, y)=1 \text { for } \\
& x \in\left\langle x_{0}-\frac{p}{2} ; x_{0}+\frac{p}{2}\right\rangle \wedge y \in\left\langle y_{0}-\frac{p}{2} ; y_{0}+\frac{p}{2}\right\rangle \\
& P(x, y)=0 \text { for } \\
& x \notin\left\langle x_{0}-\frac{p}{2} ; x_{0}+\frac{p}{2}\right\rangle \vee y \notin\left\langle y_{0}-\frac{p}{2} ; y_{0}+\frac{p}{2}\right\rangle .
\end{aligned}
$$

The parameter $p$ is the pixel size, i.e., $p=55 \mu \mathrm{m}$ in the case of the Medipix2. Graphical representations of the matrixes $G_{i}, S_{i}$ and $P$ are in Fig. 10.

The function $S_{i}(x, y)$ represents the proportion of energy which is deposited in the central pixel when a photon hits the position $(x, y)$ within the $3 \times 3$ matrix of pixels. For example if the center of the middle pixel is hit, the full energy is deposited in that pixel (a cluster of size one pixel would be detected in the Timepix). If the event occurs at the rim of the pixel, then a cluster of 2 or 3 pixels would be detected in the Timepix, and the central pixel collects only part of the deposited energy. If the photon is absorbed in $i$-th layer at position $(x, y)$ the energy detected in the central pixel is:

$$
E_{i j}^{D}(x, y)=E_{j} S_{i}(x, y),
$$

where $E_{j}$ is the $j$-th energy of the input spectrum. To generate the final detected spectrum, the energies $E_{i j}^{D}$ are calculated for all layers and positions $(x, y)$ across the $3 \times 3$ array of pixels. These numbers are binned into the calculated spectrum $\Xi(E)$ with weights:

$$
w_{i j}=\Gamma_{j} e^{-\mu_{j} \rho \frac{i-1}{L-1} d}
$$

where:

d is the thickness of the Silicon sensor $(300 \mu \mathrm{m})$,

$\Gamma_{j} \quad$ is the X-ray intensity in the $j$-th bin of the input spectrum,

$\mu_{j} \quad$ is the X-ray mass attenuation coefficient for Silicon at energy $E_{j}$. It excludes Rayleigh scattering that does not generate an electron and thus does not lead to detection of the photon.

$\rho \quad$ is the density of Silicon.

Thus, the calculation accounts for the higher likelihood of low energy photon absorption in the top portion of the detector. On the other hand, the calculation neglects photon scattering and contribution of X-ray fluorescent photons from bump-bonds connecting the sensor and readout and from other materials surrounding the sensor [10]. If the energy $E_{i j}^{D}(x, y)$ is lower than a set threshold $E_{\mathrm{TH}}$, the weight $w_{i j}$ is set to zero.

The resulting spectrum is finally convolved with electronic noise:

$$
\Xi_{\mathrm{FIN}}(E)=\int_{0}^{E} \Xi(E-\varepsilon) \frac{1}{\sqrt{2 \pi \sigma_{e}}} e^{-\frac{\varepsilon^{2}}{2 \sigma_{e}^{2}}} d \varepsilon,
$$

where $\sigma_{e}$ is the detector resolution given by the readout electronics noise and fluctuations in the charge carrier generation [10].

The parameters $\Gamma_{j}$ in (7) are determined from a fit in case that the calculation of charge sharing is used to analyze experimental data. Thus, the values resulting from the fit include also the detection efficiency of the sensor at the given energy. Hence, the (7) does not contain any extra term to correct on the detector efficiency. Nevertheless, the (7) corrects on the attenuation of photons inside the detector and therefore different numbers of photons detected along the depth of the sensor. The detection efficiency is considered in a separate step, in case that the charge sharing calculation is used to generate a detector response matrix as described later.

The advantage of this analytical approach compared to a Monte-Carlo simulation is the possibility to use the described function for fitting the measured spectra. The parameters such as $\sigma_{i=1}, \sigma_{i=L}$ and $\sigma_{e}$ can be determined from the fit and 
adjusted experimentally for cases where the sensor properties change, for example due to radiation damage of the sensor and readout or simply just by applying a different bias.

The described charge sharing calculation was implemented in the DataMaster, an in-house data acquisition and analysis software package. In Figs. 6 to 9 there are shown comparisons between the calculated and measured fluorescent X-ray spectra of various elements. The energy threshold was set to $8.5 \mathrm{keV}$. The overall energy range of the calculation corresponded to the measured spectra. The calculation used 30 layers across the detector thickness, the $3 \times 3$ array of pixels was divided for the numerical calculation into $150 \times 150$ subpixels. The input spectrum for the calculation in all cases consisted of the $K \alpha_{1}$ and $K \beta_{1}$ lines as listed in Table I.

The Bremsstrahlung photons from the X-ray tube scattered elsewhere in the X-ray cabinet were suppressed by the lead detector enclosure. Therefore, the only substantial path for these photons to enter the detector was after scattering inside the sample. The sample was chosen to be as thin as possible to reduce the scattering. There was observed no significant contribution of the scattered photons and therefore, the Bremsstrahlung background was neglected.

Intensities $\Gamma_{\mathrm{j}}$ of the $\mathrm{K} \alpha_{1}, \mathrm{~K} \beta_{1}$ lines, the parameters of the charge spread on the top and bottom of the sensor $\sigma_{i=1}, \sigma_{i=L}$ and the energy resolution $\sigma_{e}$ were set as free parameters and determined from the fits of measured data. The Fig. 11 shows dependency of the charge spread parameters on the top and bottom of the sensor $\sigma_{i=1}, \sigma_{i=L}$ and of the energy resolution $\sigma_{e}$.

The final energy resolution is $1.63 \mathrm{keV} @ 25.3 \mathrm{keV}$ (Full Width at Half Maximum of the $\mathrm{Sn} \mathrm{K} \alpha$ peak). The energy resolution published in [9] is of $1.81 \mathrm{keV} @ 25 \mathrm{keV}$ (FWHM). Researchers in [11] achieved resolution of $2.14 \mathrm{keV} @ 22 \mathrm{keV}$. The energy resolution was in all cases determined from a spectrum generated as sum of spectra of individual pixels. The good result of the energy resolution obtained here can be accounted to the per-pixel energy calibration, which reduced the effect of variation of the truth energy threshold in individual pixels (Fig. 4).

\section{DETECTOR RESPONSE MATRIX}

The charge sharing function described in the previous section was used to generate the detector response matrix. The parameters $\sigma_{i=1}=11.575 \mu \mathrm{m}, \sigma_{i=L}=1.425 \mu \mathrm{m}$, and $\sigma_{e}=0.591$ $\mathrm{keV}$ were calculated as average numbers of the values determined by fits of individual spectra (Fig. 11). The rest of the parameters were the same as for the spectrum fitting described in the previous section.

The XRF imaging discussed here was considered to be a qualitative rather than quantitative analysis therefore the detector's detection efficiency was not precisely measured and folded into the response matrix. However, the columns of the response matrix were multiplied by correction:

$$
C(E)=\frac{1-e^{-\mu(E) \rho d}}{1-e^{-\mu\left(E_{\min }\right) \rho d}}
$$

where $E_{\min }$ is the lowest energy of the response matrix, $\mu$ is the mass attenuation coefficient of Silicon, which again excludes

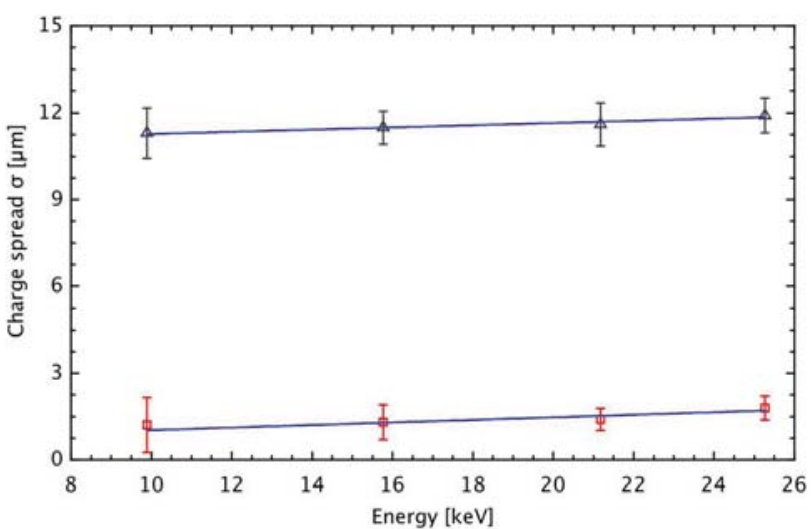

a)

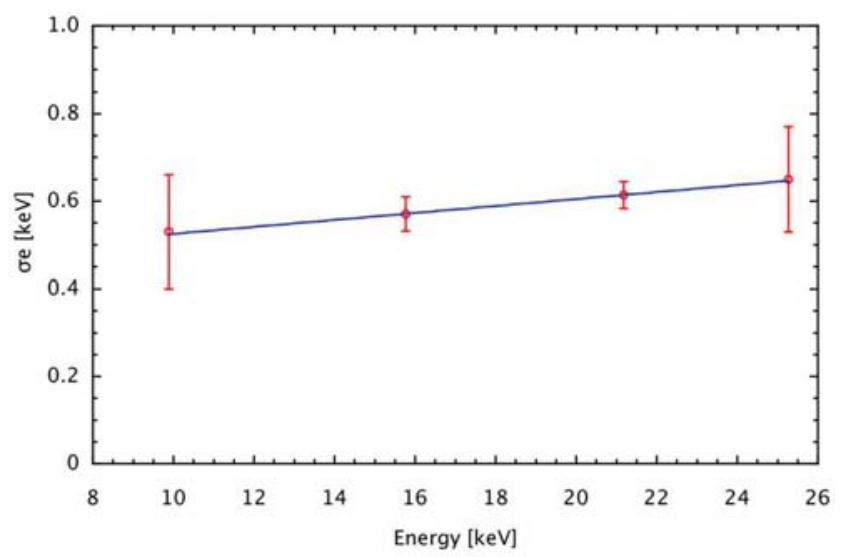

b)

Fig. 11. Dependency of the parameters: (a) $\sigma_{i=1}$ (triangles), $\sigma_{i=L}$ (squares) and (b) $\sigma_{e}$ on the X-ray energy. The parameters were obtained from the fits in Figs. 6 to 9. The data were fitted with a linear function (blue line).

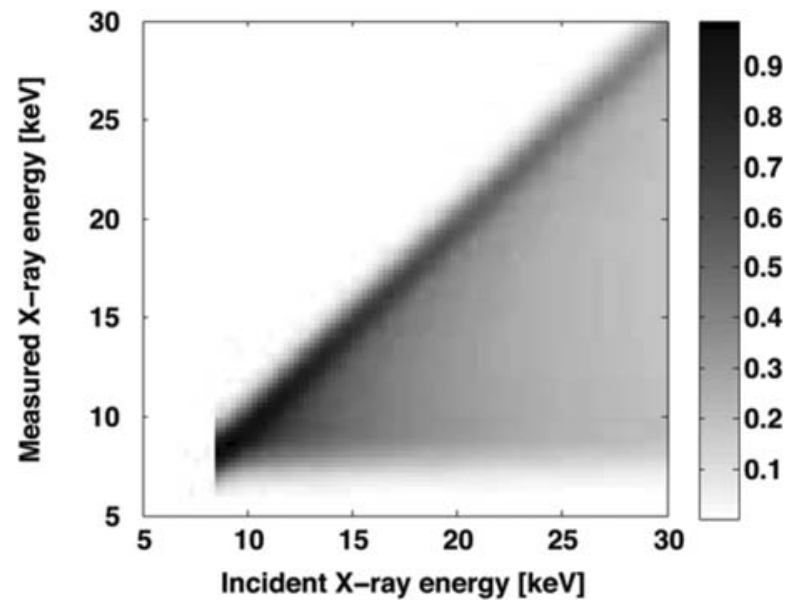

Fig. 12. The Medipix 2 detector response matrix. The matrix is truncated at energy of $8.5 \mathrm{keV}$, which is the threshold set in the measurement and the data analysis.

Rayleigh scattering. The parameter $\rho$ is the density of Silicon and $d$ is the thickness of the Medipix 2 sensor $(300 \mu \mathrm{m})$. The calculated detector response matrix is shown in Fig. 12. The matrix was of size $113 \times 113$ with energy bins from 4.75 to 33 $\mathrm{keV}(0.25 \mathrm{keV}$ per bin). 


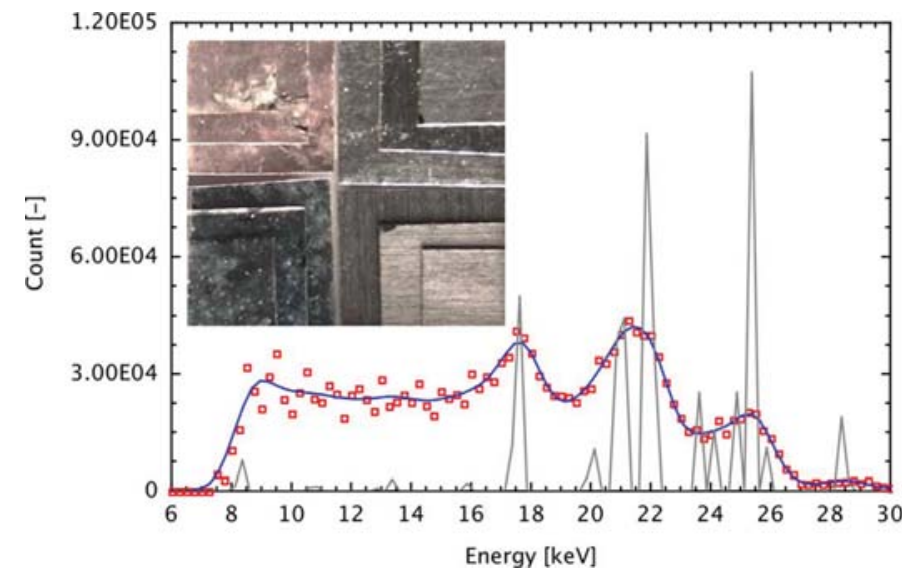

Fig. 13. Total X-ray fluorescent spectrum of the sample. The squares represent the measured data and the full line approximating the data is the least square fit using the detector response matrix. The remaining histogram line shows the "true" spectrum found by the fit (see the discussion of (10)). The inset shows a photo of the sample.

The generation of the response matrix required $\sim 5$ minutes using a $2.66 \mathrm{GHz}$ Intel Dual Core processor. Further improvement on the calculation speed should be achieved by utilization of Graphics Processing Units (GPUs) that are present in nearly all current graphics cards and through CUDA [17] or OpenCL [18] programming languages also accessible for numerical calculations. Greater calculation speed of the charge sharing would open the possibility to use the charge sharing function, for example, in the per-pixel spectra fitting during the calibration process and therefore improve the quality of the energy calibration.

\section{X-RAY FluORESCEnCE IMAGing}

The X-ray fluorescent imaging was tested on a sample consisting of four different foils of Mo, Pd, Ag and $\mathrm{Sn}$. The inset of Fig. 13 shows the sample. Each corner of the sample contains three layers of foils of the same material. The major emission lines of the elements used in the sample are listed in Table I.

The sample was measured in the geometry shown in Fig. 1. The pinhole diameter was reduced to $1 \mathrm{~mm}$. The other dimensions and exposure times were the same as that used for the energy calibration measurement. The disadvantage of the geometrical arrangement in Fig. 1, where the sample was rotated by $45^{\circ}$, was the introduction of a parallax into the XRF image. This geometry was chosen due to constraint of the current X-ray cabinet where the measurement was performed. The setup will be changed in the future to allow the XRF camera to take images perpendicular to the sample surface.

The total spectrum summed from all pixels is shown in Fig. 13.

The measured spectrum can be expressed as:

where:

$$
M \cdot \vec{r}=\vec{m}
$$

$M \quad$ is the detector response matrix,

$\vec{r} \quad$ is the incident $\mathrm{X}$-ray spectrum,

$\vec{m} \quad$ is the measured spectrum,

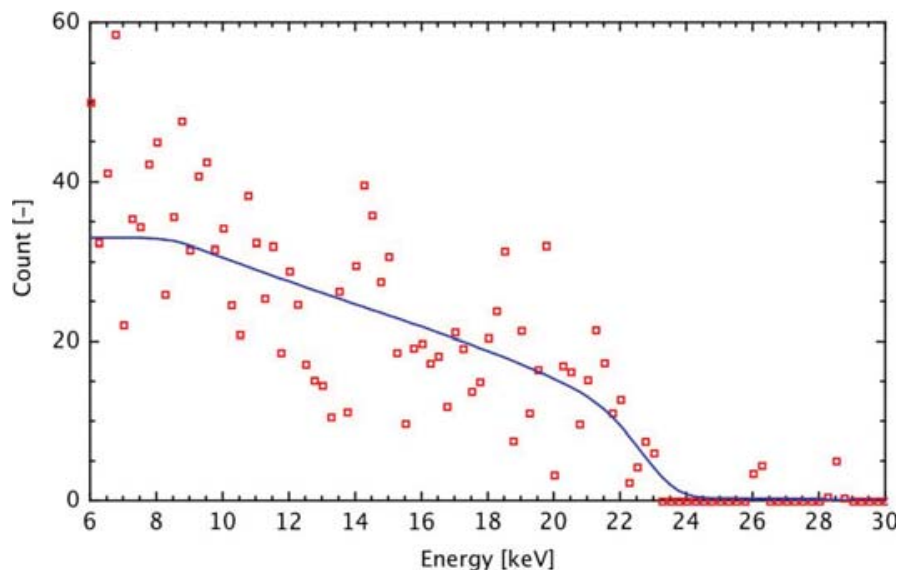

Fig. 14. Example of the fitted integral spectrum in a selected pixel.

The vector $\vec{r}$, representing the true incident spectrum was found by solving (10) using the LSQNONNEG function provided by Matlab [19]. The LSQNONNEG function implements the linear least square method with non-negativity constraints, i.e., $\mathrm{X}=\mathrm{LSQNONNEG}(\mathrm{C}, \mathrm{d})$ returns the vector $\mathrm{X}$ that minimizes $\mathrm{NORM}(\mathrm{d}-\mathrm{C} * \mathrm{X})$ subject to $\mathrm{X}>=0$. It is suitable function for this problem, as negative values would have no physical meaning. The fit was performed on the total integral spectrum, which was then differentiated. The result is shown in Fig. 13. Most of the X-ray emission energies listed in Table I were found in the spectrum. However, the $\mathrm{K} \alpha_{1}$ and $\mathrm{K} \alpha_{2}$ energies are closer together than the bin width of $0.25 \mathrm{keV}$ and therefore were not distinguished. The $\mathrm{K} \beta_{1}$ lines, and in the case of $\mathrm{Pd}$ also the $\mathrm{K} \beta_{2}$ line were also resolved in the spectrum. The line found just below $26 \mathrm{keV}$ is most likely the $\mathrm{K} \beta_{2}$ line of $\mathrm{Ag}$, incorrectly reconstructed at higher energy due to systematic errors in the per-pixel energy calibration. The noise in the spectrum was very likely responsible for the peaks below $17 \mathrm{keV}$.

The total spectrum was used to define constraints for the perpixel analysis. The integral spectra in individual pixels were also fitted using the non-negative least squares method. The counting statistics in individual pixels were very low (see Fig. 14). Therefore, the number of free parameters in the least square fit had to be restricted and the response matrix was limited to columns appropriate for the $K \alpha_{1}$ and $K \beta_{1}$ lines of the elements otherwise identified in the total spectrum. No other constrains were applied.

The resulting fitted spectra in each pixel were analyzed by checking energy windows where the $K \alpha_{1}$ lines of elements present in the sample were expected. These windows were selected based on the fit of the total spectrum (Fig. 13). The energy windows were: $16.0<\mathrm{E}_{\mathrm{Mo}}<19.0 \mathrm{keV}, 21.5<\mathrm{E}_{\mathrm{Pd}}<22.7$ $\mathrm{keV}, 22.7<\mathrm{E}_{\mathrm{Ag}}<24 \mathrm{keV}$ and $24<\mathrm{E}_{\mathrm{Sn}}<25 \mathrm{keV}$. An additional condition on the amplitude of the peak within the energy window was applied. The amplitude had to be larger than amplitude of peaks in the other windows for the given pixel. Only that element for which all of these conditions were fulfilled was allocated to the examined pixel. Another possibility would be to associate the peak intensity to the concentration of an appropriate element. This approach would be suitable for mixed materials. However, it was not used in this 


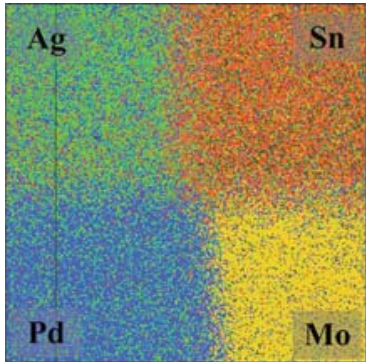

Fig. 15. XRF image of the sample from Fig. 13 with colours representing the identified elements (green: Ag, red: Sn, blue: Pd, yellow: Mo).

demonstration of the XRF imaging technique. The resulting material-resolved image is shown in Fig. 15 where each color represents the element found in the spectrum of the given pixel. Ag was correctly found in $52 \%$ of pixels where it should be observed, Sn in $59 \%$, Pd in $76 \%$ and Mo in $84 \%$ of pixels.

\section{CONCLUSION}

An application of the Medipix2 to X-ray fluorescence imaging was presented. The per-pixel energy calibration of the detector was described. The effect of charge sharing between pixels was discussed and the numerical approach to its calculation was explained. The results of charge sharing calculations were compared with measured X-ray fluorescent spectra of Ge, $\mathrm{Zr}$, Pd and $\mathrm{Sn}$, and good agreement was found.

The charge sharing calculation was used to generate a Medipix 2 detector response matrix that was subsequently used for the analysis of the X-ray fluorescence emission spectrum of a sample consisting of Mo, Pd, Ag and Sn foils. All elements were resolved by the per-pixel spectrum analysis in both the energy and spatial domain.

The particular Medipix2 device used for the discussed experiments has been used in the past for a great variety of measurements not only with X-rays, but also with fast neutrons and ions. Therefore, some radiation damage has already occurred. However, the damage is not exactly characterized. Regardless, excellent results of the detector charge sharing characterisation were achieved. That only underlines advantages of the presented charge sharing calculation technique, since the parameters for the charge sharing function can be easily determined experimentally and thus adjusted during the detector lifetime.

The new generation of imaging detectors Medipix 3 contains electronics that treats the charge sharing effect within the pixel readout by summing the charge deposited in neighbouring pixels [20]. Therefore, the spectral response of the Medipix3 should be significantly improved. However, the latest results indicate that there would still be a need for the charge sharing characterisation because the charge sharing may not be entirely suppressed by the readout electronics [21]. Moreover, the Medipix2 device will still remain a useful tool for a number of applications. Thus, a precise and rapid charge sharing characterisation remains an important issue.

\section{ACKNOWLEDGMENT}

This work was carried out in the framework of the CERN Medipix Collaboration.

\section{REFERENCES}

[1] C. G. Ryan, R. Kirkham, R. M. Hough, G. Moorhead, D. P. Siddons, M. D. de Jonge, D. J. Paterson, G. De Geronimo, D. L. Howard, and J. S. Cleverley, "Elemental X-ray imaging using the Maia detector array: The benefits and challenges of large solid-angle," Nucl. Instrum. Methods Phys. Res. A, vol. 619, pp. 37-43, 2010.

[2] Z. W. Chen, W. M. Gibson, and H. Huang, High Definition X-Ray Fluorescence: Principles and Techniques. New York: Hindawi Publishing Corp., vol. 2008, X-Ray Optics and Instrumentation, Article ID 318171.

[3] P. Bruyndonckx, A. Sasov, and X. Liu, "Laboratory 3D micro-XRF/ micro-CT imaging system," in Proc. 10th Int. Conf. X-ray Microscopy, 2011, pp. 61-64, AIP Conf. 1365.

[4] X. Llopart, M. Campbell, R. Dinapoli, D. San Segundo, and E. Pernigotti, "Medipix2: A 64-k pixel readout chip with 55- $\mu \mathrm{m}$ square elements working in single photon counting mode," IEEE Trans. Nucl. Sci., vol. 49, no. 5, pp. 2279-2283, Oct. 2002.

[5] R. Aamir, S. P. Lansley, R. Zainon, M. Fiederle, A. Fauler, D. Greiffenberg, P. H. Butlera, and A. P. H. Butler, "Pixel sensitivity variations in a CdTe-Medipix2 detector using poly-energetic x-rays," J. Instrum. 2011, 6 C01059 doi:10.1088/1748-0221/6/01/C01059.

[6] V. Tichy, T. Holy, J. Jakubek, V. Linhart, S. Pospisil, and Z. Vykydal, "X-ray fluorescence imaging with pixel detectors," Nucl. Instrum. Methods Phys. Res. A, vol. 591, pp. 67-70, 2008.

[7] M. Fiederle, D. Greiffenberg, J. Idarraga, J. Jakubek, V. Kral, C. Lebel, C. Leroy, G. Lord, S. Pospisil, V. Sochor, and M. Suk, "Energy calibration measurements of MediPix2," Nucl. Instrum. Methods Phys. Res. $A$, vol. 591, pp. 75-79, 2008.

[8] National Institute of Standards and Technology [Online]. Available: http://www.nist.gov

[9] C. Ponchut, "Correction of the charge sharing in photon-counting pixel detector data," Nucl. Instrum. Methods Phys. Res. A, vol. 591, pp. 311-313, 2008.

[10] A. Korn, M. Firsching, G. Anton, M. Hoheisel, and T. Michel, "Investigation of charge carrier transport and charge sharing in X-ray semiconductor pixel detectors such as Medipix2," Nucl. Instrum. Methods Phys. Res. A, vol. 576, pp. 239-242, 2007.

[11] T. Michel, P. T. Talla, M. Firsching, J. Durst, M. Bohnel, and G. Anton, "Reconstruction of X-ray spectra with the energy sensitive photon counting detector Medipix2," Nucl. Instrum. Methods Phys. Res. A, vol. 598, pp. 510-514, 2009.

[12] J. Jakubek, J. Dammer, T. Holy, M. Jakubek, S. Pospisil, V. Tichy, J. Uher, and D. Vavrik, "Spectrometric properties of TimePix pixel detector for X-ray color and phase sensitive radiography," in Proc. IEEE Nuclear Science Symp. Conf. Rec., 2007.

[13] X. Llopart, R. Ballabriga, M. Campbell, L. Tlustos, and W. Wong, "Timepix, a $65 \mathrm{k}$ programmable pixel readout chip for arrival time, energy and/or photon counting measurements," Nucl. Instrum. Methods Phys. Res. A, vol. 581, pp. 485-494, 2007.

[14] J. Jakubek, "Semiconductor pixel detectors and their applications in life sciences," in Proc. Pixel Int. Workshop, Batavia, IL, Sep. 23-26, 2008

[15] H. G. Spieler and E. E. Haller, "Assessment of present and future largescale semiconductor detector systems " IEEE Trans. Nucl. Sci., vol. 32, no. 1, pp. 419-426, Feb. 1985.

[16] J. Uher, G. Roach, and J. Tickner, "Gamma-ray spectrum fitting and peak identification tools for Matlab and Java," Nucl. Instrum. Methods Phys. Res. A, vol. 619, no. 1-3, pp. 457-459, 2010.

[17] NVidia [Online]. Available: http://www.nvidia.com/object/what_is_cuda_new.html

[18] OpenCL [Online]. Available: http://www.khronos.org/opencl

[19] MATLAB ${ }^{\text {TM }}$ [Online]. Available: http://www.mathworks.com

[20] R. Ballabriga, M. Campbell, E. H. M. Heijne, X. Llopart, and L. Tlustos, "The Medipix3 prototype, a pixel readout chip working in single photon counting mode with improved spectrometric performance," in Proc. IEEE Nuclear Science Symp. Rec., 2006.

[21] P. T. Talla, P. Bartl, T. Michel, J. Durst, and G. Anton, "A modified spectrum reconstruction method for the charge summing mode of Medipix3," Nucl. Instrum. Methods Phys. Res. A, submitted for publication. 